Z. Epileptol. 2021 · 34:396-402

https://doi.org/10.1007/s10309-021-00441-0

Angenommen: 24. August 2021

Online publiziert: 28. September 2021

(c) Der/die Autor(en) 2021

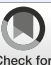

\section{Akut symptomatische Anfälle bei Neonaten und Einsatz des amplitudenintegrierten EEGs (aEEG)}

\author{
Katrin Klebermass-Schrehof \\ Abteilung für Kinder- und Jugendheilkunde, Division für Neonatologie, pädiatrische Intensivmedizin und \\ Neuropädiatrie, Medizinische Universität Wien, Wien, Österreich
}

\section{Zusammenfassung}

\section{In diesem Beitrag}

- Indikationen zum kontinuierlichen Monitoring

- Einsatz des aEEG bei neonatalen Anfällen

- Haben Anfälle beim Neugeborenen einen Einfluss auf das spätere entwicklungsneurologische Outcome?

- Automatische Anfallserkennung - Zukunftsmusik oder schon Realität?

- Limitationen und Vorteile des aEEGs bei neonatalen Anfällen

Hintergrund: Anfälle beim Neugeborenen sind in den allermeisten Fällen Symptom einer zugrunde liegenden neurologischen Störung im Sinne von „akut symptomatischen Anfällen“. Die Diagnose von neonatalen Anfällen erfolgt laut ILAE (International Liga Against Epilepsy) nur über die Elektroenzephalografie, und erst dann wird beschrieben, ob die Anfälle von klinischen Symptomen begleitet werden oder nicht. Somit hat sich in den letzten Jahren die vereinfachte Methode des amplitudenintegrierten EEGs (aEEG) als direkt am Krankenbett einsetzbare Methode zum Langzeitmonitoring als hilfreich erwiesen und stellt nicht nur eine „kompensatorische Maßnahme“ bis zur Verfügbarkeit eines konventionellen EEGs dar. Ziel der Arbeit: In diesem Artikel wird der Einsatz des aEEGs bei neonatalen Anfällen diskutiert.

Ergebnisse und Diskussion: Zur genaueren Erfassung der "seizure burden“ (Anfallsdauer über einen definierten Zeitraum) und zur Therapiesteuerung stellt das aEEG neben dem konventionellen Video-EEG eine hilfreiche zusätzliche Methode dar. Die Dauer und Häufigkeit der Anfälle, die Hintergrundaktivität und die Erfahrung des Befunders spielen bei der Erkennung von Anfällen im aEEG eine entscheidende Rolle. Nur kurz dauernde, fokale oder sich nur wenig von der Grundaktivität unterscheidende Anfälle können weniger gut erfasst werden, wobei länger dauernde Anfälle, die sich regional ausbreiten von einem erfahrenen Befunder in bis zu $85 \%$ auch mittels aEEG erkannt werden können. Da es zunehmend Daten gibt, dass die "seizure burden" mit dem entwicklungsneurologischen Outcome korreliert, ist eine möglichst kontinuierliche elektrophysiologische Überwachung dieser Patienten anzustreben. Hier kann das aEEG einen wichtigen Beitrag leisten.

\title{
Schlüsselwörter
}

Anfälle beim Neugeborenen · Neonatale Anfälle · Neurophysiologische Überwachung am Krankenbett · Langzeit-EEG-Monitoring

\section{Hintergrund}

Anfälle beim Neugeborenen sind in den allermeisten Fällen Symptom einer zugrunde liegenden neurologischen Störung im Sinne von "akut symptomatischen Anfällen". Im Jahr 2021 hat die ILAE (International League Against Epilepsy) eine neue Klassifikation von zerebralen Anfällen beim Neugeborenen vorgestellt [18], die die Rolle der Elektroenzephalographie zur Diagnose von Anfällen in dieser Altersgruppe unterstreicht. Die Definition von Anfällen erfolgt hierbei nur über die Elektroenzephalographie (EEG), und erst danach wird beschrieben, ob die Anfälle von klinischen Symptomen begleitet werden oder nicht (dann werden sie als "rein elektrographische Anfälle" bezeichnet). Somit ist eine nur auf der Klinik basierende Diagnose von zerebralen Anfällen beim Neugeborenen nicht mehr empfohlen, da dies auf der einen Seite zur Überdiagnose bzw. Übertherapie, andererseits zum verminderten 


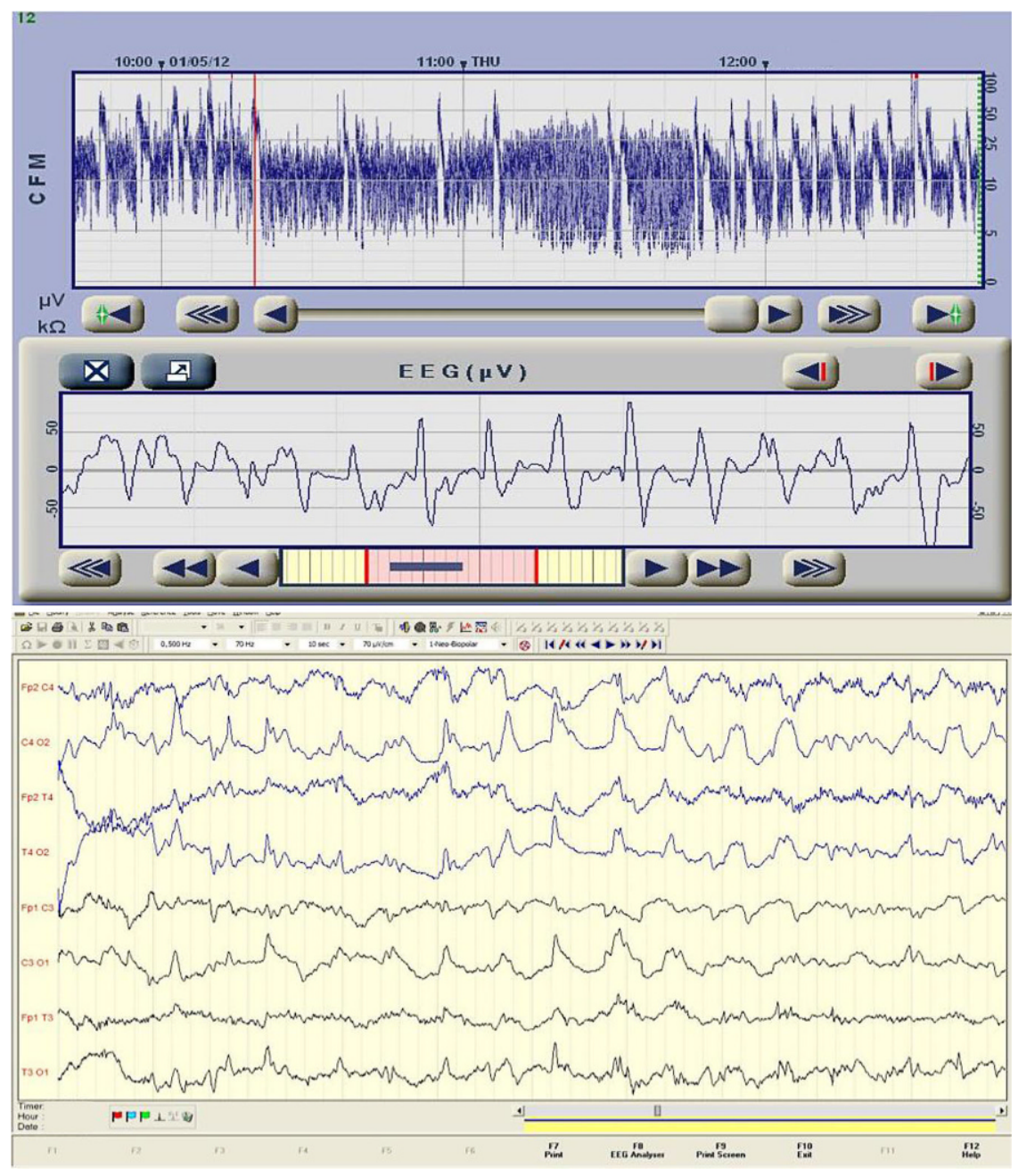

Abb. $1 \Delta$ Ableitung eines ehemaligen Frühgeborenen der 24 +3 SSW im korrigierten Alter von $35+2$ SSW nach dem Auftreten von Anfällen im Rahmen einer Hypoglykämie. Grundaktivität: kontinuierliches Grundmuster mit altersentsprechender Amplitudenhöhe, im ruhigen Schlaf noch diskontinuierliches Hintergrundmuster (dies dem korrigierten Alter entsprechend), erkennbare Schlaf-Wach-Zyklen und repetitive Anfälle (speziell zwischen 9:30-10:20 und 11:50-12:20 sogar im Sinne eines Status epilepticus). Im Roh-EEG (zum Zeitpunkt der roten Markierung) rhythmische Sharpwave-Aktivität; aEEG Ableitung, Messgerät und Software (Viewer) Olympic CFM6000, Natus Medical Incorporated, Pleasanton, CA, USA. Im unteren Bild das konventionelle EEG desselben Patienten im Rahmen eines weiteren Anfalls. Die obere Kurve zeigt das amplitudenintegrierte EEG (des P3/P4Kanals) mit einer $x$-Achse $1 \mathrm{~cm}=10 \mathrm{~min}$ und einer $Y$-Achse in $\mu \mathrm{V}$ und linear bis $10 \mu \mathrm{V}$ und darüber semilogarithmischer Aufzeichnung. Die untere Kurve zeigt das Roh-EEG (des P3/P4-Kanals) mit einer $x$-Achse $1 \mathrm{~cm}=1$ s und einer Y-Achse in $\mu \mathrm{V}$ (linear) (so wie auch im darunter liegenden konventionellen EEG-Bild mit 8 Kanälen); BrainQuick EEG-Software and viewer, Micromed Group, Treviso, Italien

Erfassen von Anfällen führen kann [20]. Da angenommen wird, dass eine fehlende Behandlung bzw. ein Nichterkennen von Anfällen zum Triggern („,kindling ${ }^{\prime \prime}$ ) von weiteren Anfällen und zur Verschlechterung einer vorliegenden Hirnschädigung führen kann [30], ist eine möglichst genaue Anfallserkennung (inklusive genaue Dauer) beim Neugeborenen anzustreben. Auf der anderen Seite kann eine Überbehandlung durch eine unnötige medikamentöse Therapie mit an sich neuro- coupling", bei dem elektrographische Anfallstätigkeit weiterbesteht, obwohl sich die klinische Symptomatik bessert bzw. verschwindet [23].

Die häufigsten Ursachen von zerebralen Anfällen beim Neugeborenen sind hypoxisch-ischämische Läsionen (mit fast 70\%) inklusive neonataler arterieller und venöser Infarkte, gefolgt von intrakraniellen Blutungen (intraventrikuläre Blutungen [IVH] beim Frühgeborenen, Subarachnoidalblutung beim reifen Neugeborenen), Infektionen und Stoffwechselveränderungen (von einfacher Hypoglykämie bis zur komplexen angeborenen Stoffwechselerkrankung). Auch angeborene zerebrale Fehlbildungen, genetische Syndrome oder Drogen/Medikamente können beim Neugeborenen Anfälle auslösen. Die Diagnostik von Anfällen beim Neugeborenen und Frühgeborenen ist schwierig und eine kontinuierliche neurophysiologische Überwachung ist dabei unabdingbar. In nur wenigen, sehr spezialisierten Abteilungen ist eine kontinuierliche bzw. langfristigere Überwachung mittels konventionellen, mehrkanaligen EEGs inklusive Videoüberwachung möglich, auch wenn dies die anzustrebende bzw. wünschenswerte Methodik darstellt. Somit hat sich in den letzten Jahren die vereinfachte Methode des amplitudenintegrierten EEGs (aEEG) als zeitkomprimierte, direkt am Krankenbett interpretierbare Methode als hilfreich erwiesen und stellt nicht nur eine "kompensatorische Maßnahme" bis zur Verfügbarkeit eines konventionellen EEGs dar [4]. Bereits in den 60er-Jahren von Maynard et al. entwickelt [15], wurde das amplitudenintegrierte EEG initial zur Überwachung nach Trauma, Operation oder ischämischem Insult auf der Intensivstation von Erwachsenen angewandt, hat aber seit den 80er-Jahren v. a. auf neonatologischen Intensivstationen Verbreitung gefunden. Heutzutage werden digitale, mehrkanalige Varianten verwendet (meist P3-P4, C3-P3, C4-P4, gemeinsam mit dem zugrunde liegenden RohEEG und der kontinuierlich aufgezeichneten Elektrodenimpedanz). Moderne aEEGMonitore verfügen auch über einen automatischen Seizure-detection-Algorithmus zur weiteren Vereinfachung, wobei aber eine klinische, visuelle Überprüfung der markierten Episoden immer empfohlen 


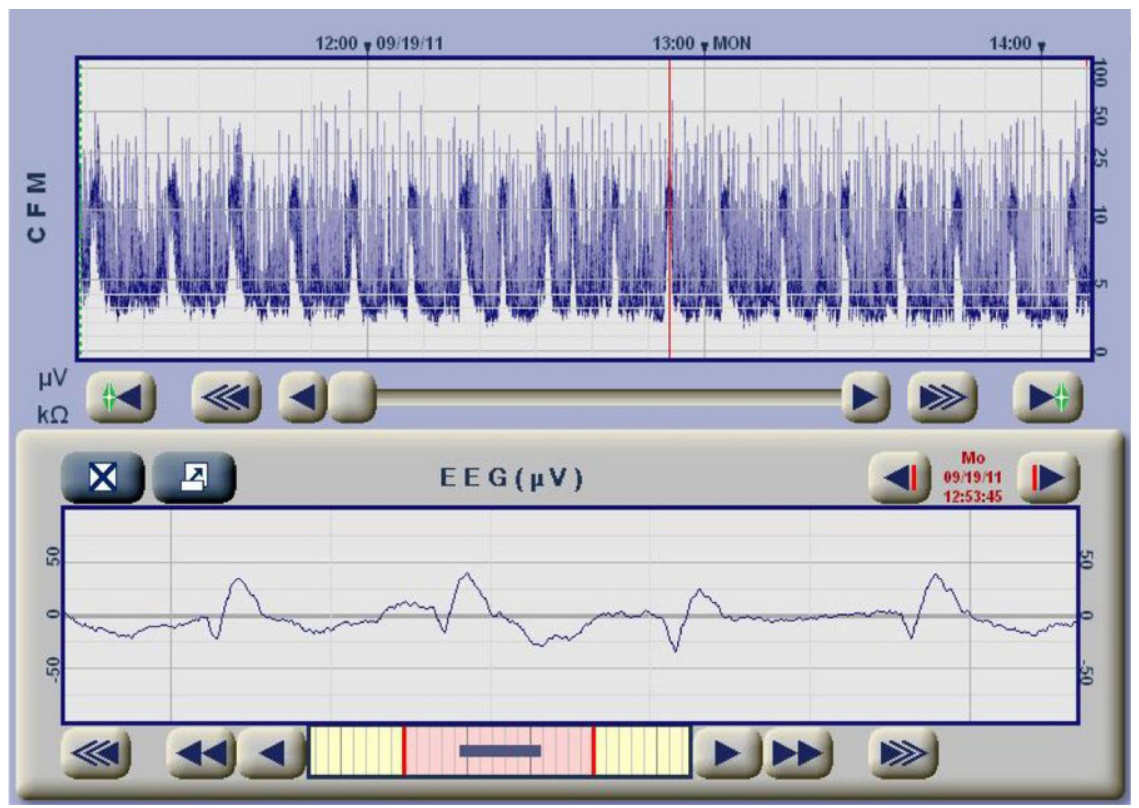

Abb. 2 \ Status epilepticus im Sinne eines „Sägezahnmusters" im aEEGund Sharp-wave-Sequenzen im korrespondierenden Roh-EEG. Die obere Kurve zeigt das amplituden-integrierte EEG (des P3/P4Kanals) mit einer $x$-Achse $1 \mathrm{~cm}=10$ min und einer $Y$-Achse in $\mu V$ und linear bis $10 \mu V$ und darüber semilogarithmischer Aufzeichnung. Die untere Kurve zeigt das Roh-EEG (des P3/P4-Kanals) mit einer $\mathrm{x}$-Achse $1 \mathrm{~cm}=1$ s und einer Y-Achse in $\mu \mathrm{V}$ (linear) (so wie auch im darunter liegenden konventionellen EEG-Bild mit 8 Kanälen); aEEG Ableitung, Messgerät und Software (Viewer) Olympic CFM6000, Natus Medical Incorporated, Pleasanton, CA, USA

ist [14]. Aufgrund der in Zeit und Region komprimierten Darstellung ist eine - im Vergleich zum konventionellen EEG reduzierte Möglichkeit der Anfallserkennung zu erwarten. Eine Schulung in der aEEG-Interpretation ist empfohlen, und eine Differenzierung von Artefakten kann schwierig sein.

Wie sich in letzter Zeit zunehmend herausstellt, ist die genaue Erfassung der "seizure burden" (genaue Dauer von Anfallsaktivität in einem definierten Zeitraum) für die Prognose wichtig, und auch Therapien lassen sich mit einer kontinuierlichen Überwachung genauer steuern. Erste Daten zeigen, dass die exakte "seizure burden" mit dem Langzeitoutcome und der Läsionsschwere im MRT korreliert [26]. Daher empfehlen internationale Guidelines $[6,24]$ eine kontinuierliche elektrophysiologische Überwachung an der neonatologischen Intensivstation, jedoch fehlen klare Empfehlungen, wie konventionelle EEG und aEEG-Ableitungen im klinischen Setting kombiniert werden sollen. Als Goldstandard zur Anfallsdiagnostik wird das konventionelle EEG inklusive Videoüberwachung angestrebt, aber es wird emp- fohlen, dies mit einer bihemisphärischen aEEG-Trendüberwachung zu ergänzen.

Neugeborene, die ein besonders hohes Risiko für eine akute Schädigung des Gehirns haben, haben auch ein besonders hohes Risiko, Anfälle zu entwickeln, und sollten somit möglichst kontinuierlich überwacht werden. Es handelt sich dabei um Kinder mit angeborenen Herzfehlern, an der extrakorporalen Membranoxygenierung oder schwerer respiratorischer Problematik (persistierende pulmonale Hypertension des Neugeborenen, Mekoniumaspirationssyndrom, schweres Respiratory-distress-Syndrom, mit Infektionen des Zentralnervensystems oder angeborenen Stoffwechseldefekten, extrem unreife bzw. instabile Frühgeborene) Schädigung aufweisen (Kinder mit hypoxisch-ischämischer Enzephalopathie [HIE]/ Asphyxie, ischämischem oder hämorrhagischem Insult, Trauma, intrakranieller/ intraventrikulärer Blutung oder einer das Gehirn mitbetreffenden genetischen Er-

\section{Indikationen zum kontinuierlichen Monitoring} oder Kinder, die bereits eine cerebrale krankung) [24]. Auch der Erfolg einer Therapie bzw. ein Ausschleichen einer Therapie sollte, wenn möglich, kontinuierlich überwacht werden [24].

\section{Einsatz des aEEG bei neonatalen Anfällen}

Anfälle beim Neugeborenen sind meist mit einer Dauer von mehr als 10 s definiert, entstehen sehr häufig zentrotemporal bzw. zeigen eine regionale Ausbreitung in diesem Bereich. Nur das Auftreten von klinischen Symptomen (seien sie motorisch oder nichtmotorisch) unterscheiden rein elektrographische Anfälle von klinisch manifesten Anfällen.

Im aEEG zeigen sich Anfälle durch einen abrupten Anstieg der Ableitungskurve (Ober- und Unterrand) mit nachfolgendem Abfall. Somit zeigt sich ein "sägezahnartiges Muster" („saw-tooth-pattern“) bei wiederholten Anfällen (• Abb. 1, 2 und 3).

In der Literatur werden verschieden hohe Anfallserkennungsraten für das aEEG angegeben. Die Dauer und Häufigkeit der Anfälle, die Hintergrundaktivität und die Erfahrung des Befunders spielen hierbei eine entscheidende Rolle.

In einer Studie von Shellhaas et al. [25] können $78 \%$ der Anfälle bei Neugeborenen über $\mathrm{C} 3 / \mathrm{C} 4$ im konventionellen EEG gesehen werden, nach einer Umwandlung dieses Kanals in ein aEEG konnten 25-60\% dieser Anfälle von Neonatologen erkannt werden. Laut einer Studie von Kadivar et al. [10] können 68-85\% Anfälle mittels aEEG (über zentral/parietal) erkannt werden. Nur sehr kurze oder nicht in der Nähe der aEEG-Elektroden gelegene Anfälle konnten weniger häufig entdeckt werden. Somit wird das aEEG als sehr gutes Werkzeug zur Anfallserkennung beim Neugeborenen angesehen, auch wenn zur finalen Diagnose und zum therapeutischen Management das konventionelle EEG inklusive Video als Goldstandard empfohlen wird.

Daten von Rennie et al. [21] zeigen, dass auch "non-experts" mittels aEEG 40-60\% der neonatalen Anfälle erkennen. Eine andere prospektive Studie allerdings - zur Anfallserkennung mittels aEEG nach kurzer Einschulung - zeigte nur eine moderate Übereinstimmung eines kurzfristig geschulten Pflegepersonals mit aEEG-Ex- 


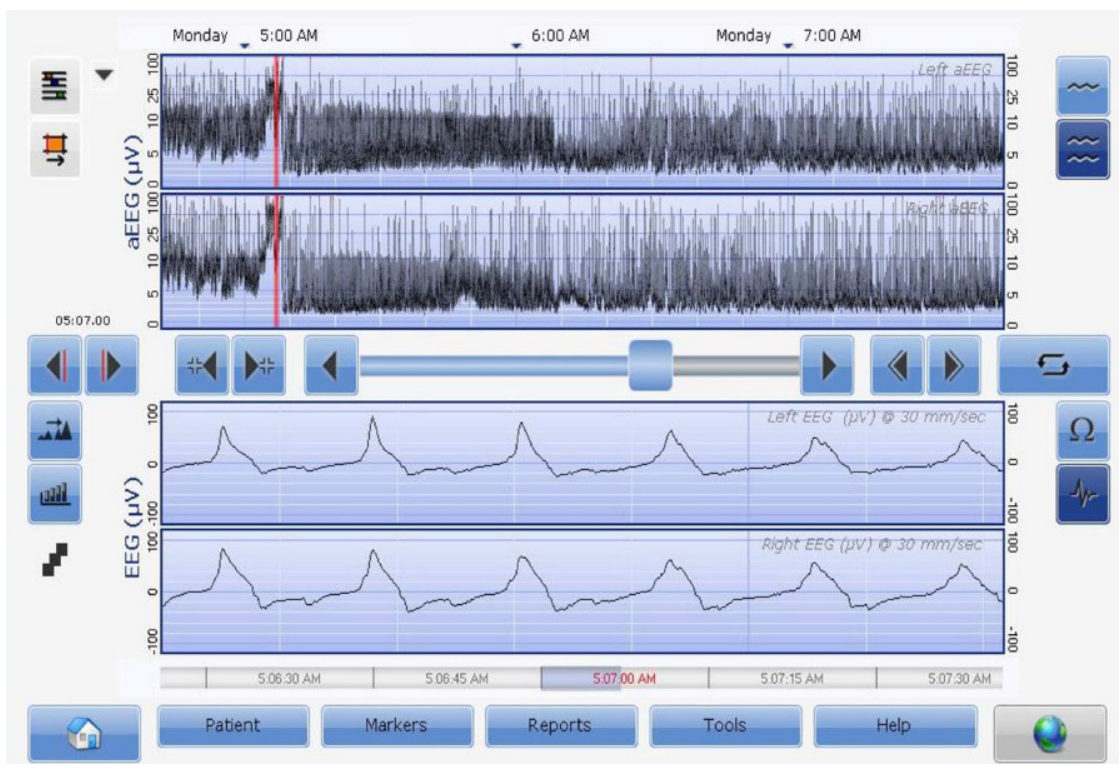

Abb. 3 ॥ Einzelner Anfall (im Bereich der roten Markierung) - sägezahnartige Hebung im aEEGmit korrespondierender Sharp-wave-Sequenz im Roh-EEG - in einer sonst abgeflachten, aber symmetrischen Hintergrundaktivität (diskontinuierliches Muster, Burst-Suppression-Muster); Olympic Brainz Monitor (OBM), Natus Medical Incorporated, Pleasanton, CA, USA

perten [3]. Nach einem kurzen Training zeigte Pflegepersonal in dieser Studie von Du Pont-Thibodeau et al. eine Sensitivität von $77 \%$ in der Erkennung von Anfällen [8]. Rakshasbhuvankara et al. [22] analysierten, dass die "interrater variability" von aEEG zur Erkennung von neonatalen Anfällen nur "ausreichend“ (kappa 0,37) für das Erkennen jedes einzelnen Anfalls, „mittelmäßig“ (kappa 0,46) für das Erkennen der individuellen Anfallsdauer, aber „beachtlich" (kappa 0,61) für das Erkennen eines Kindes mit Anfällen war, sodass auch hiermit das Potenzial des aEEGs zur Identifizierung von Kindern mit Anfällen bzw. von Kinder, die von einer längeren Video-EEG-Überwachung profitieren, unterstrichen werden kann. In einer weiteren Analyse derselben Arbeitsgruppe wurde allerdings gezeigt, dass eine medikamentöse Therapie von neonatalen Anfällen, die sich nur auf eine klinische Beobachtung oder Diagnostik mit aEEG stützt, häufig zu einer inadäquaten oder unnötigen Behandlung mit antikonvulsiver Medikation führt [19]. Entgegen initialer Befürchtungen konnten Bashir et al. bei Kinder nach HIE eine signifikante Reduktion des Antikonvulsivaeinsatzes nach Implementieren von kontinuierlicher EEG-Überwachung zeigen im Gegensatz zur Behandlung, basierend auf einem intermittieren- den Kurzzeit-EEG in Kombination mit rein klinischer Diagnostik [2]. In einer Studie, die mittels mathematischer Modelle die Sensitivität und Spezifität zur Erkennung von Anfällen des aEEGs analysiert hat, zeigte sich eine eindeutige Abhängigkeit vom Erfahrungsgrad (Experte $85 \%$, unerfahrenes Personal $40-50 \%$ ) und der zugrunde liegenden Häufigkeit und Erkennbarkeit der Anfälle (z. B. deutliche bessere Erkennbarkeit bei Kindern mit HIE im Vergleich zum Frühgeborenen) [11].

Stevenson et al. konnten zeigen, dass eine Reduktion der Elektrodenanzahl von 8 auf 4 nur zu einer minimalen Reduktion der Anfallserkennung führt ( $89 \%$ vs. $96 \%)$ [27].

\section{Haben Anfälle beim Neugebore- nen einen Einfluss auf das spätere entwicklungsneurologische Outcome?}

Da Anfälle einen Einfluss auf autonome Veränderungen (Sauerstoffverbrauch, Apnoen, RR-Schwankungen) und auch auf die Synaptogenese haben, und eine fehlende Behandlung zum Triggern („kindling“) von weiteren Anfällen und zur Verschlechterung einer vorliegenden Hirnschädigung führen kann [30], erscheint ein Einfluss von Anfällen auf das entwicklungsneuro- logische Outcome und auf das Auftreten einer postneonatalen Epilepsie sehr wahrscheinlich. Toet et al. berichten von einer Inzidenz an postneonataler Epilepsie zwischen 10 und $20 \%$ [28]. Chen et al. identifizierten eine hohe Inzidenz an elektrographischen Anfällen bei Neugeborenen mit HIE. Wiederholte Anfälle oder sogar ein Status epilepticus in den ersten $24 \mathrm{~h}$ nach Wiedererwärmung nach Hypothermiebehandlung korrelierten mit dem Auftreten einer Epilepsie nach 12 Monaten [5]. Risikofaktoren für ein ungünstiges Outcome inkludierten - in einer Analyse von Glass et al. [9] - v. a. Frühgeburtlichkeit, niedrige Apgar-Scores, niedrigen $\mathrm{pH}$ am ersten Lebenstag, Anfallsbeginn $<24 \mathrm{~h}$ oder $>72 \mathrm{~h}$ nach der Geburt, das Auftreten eines Status epilepticus, auffälligen neurologischen Status, auffällige Hintergrundaktivität im EEG und das Vorliegen einer Hirnschädigung (v.a. der Basalganglien oder des Hirnstamms). Die exakte "seizure burden" konnte mit dem Langzeitoutcome und der Läsionsschwere im MRT korreliert werden [26]. Kharoshankaya et al. berichten, dass eine hohe "seizure burden“ bei Kindern mit HIE signifikant mit einem auffälligen Outcome nach 24 und 48 Monaten korreliert, unabhängig von der ursprünglich zugrunde liegenden Schwere der HIE oder der Behandlung mit Hypothermie [12]. Auch Weeke et al. zeigten, dass das entwicklungsneurologische Outcome mit 2 Jahren (inklusive Epilepsie) mit der "seizure burden" von reifen Kinder mit HIE korreliert [29].

\section{Automatische Anfallserkennung - Zukunftsmusik oder schon Realität?}

In einer großen multizentrischen Studie wurde ein Algorithmus zur Anfallserkennung im kontinuierlichen konventionellen EEG eingesetzt und eine Sensitivität von $81 \%$ im Vergleich zu $89 \%$ in der Gruppe ohne Algorithmus gefunden. In sehr erfahrenen Zentren konnte somit kein Benefit einer zusätzlichen automatisierten Anfallserkennung nachgewiesen werden, was aber möglichweise in unerfahreneren Zentren anders sein könnte, aber weitere Studien erst noch beweisen müssten [17]. Lawrence et al. untersuchten in einer Pilotstudie den Einsatz eines Anfallserkennungsal- 
gorithmus bei kontinuierlich durchgeführtem aEEG, und hier zeigte der Algorithmus eine Anfallserkennungsrate von 55\% gesamt und $73 \%$ für Anfälle, die länger als 30 s gedauert haben [13]. In einer weiteren Studie, die verschiedene Algorithmen vergleicht, erzielten die Algorithmen zwischen 37 und $92 \%$ Anfallserkennung, im Vergleich zu 74\% Erkennung im aEEG durch Experten [7]. Apers et al. konnten aber aufjeden Fall zeigen, dass eine schnellere Therapie (innerhalb $1 \mathrm{~h}$ ) von Anfällen beim Neugeborenen erfolgte, wenn die Anfälle klinisch sichtbar waren oder ein Seizure-Detection-Algorithmus eingesetzt wurde [1].

\section{Limitationen und Vorteile des aEEGs bei neonatalen Anfällen}

Zum Erstscreening bei klinischen Auffälligkeiten und zur Therapieüberwachung kann das aEEG - ergänzend bzw. bis zur Verfügbarkeit des konventionellen EEGs - gut eingesetzt werden. Besonders die leicht übersichtliche Langzeitüberwachung, die einfache Handhabung direkt am Krankenbett und die reduzierte Elektrodenzahl sind wichtige Vorteile der Methode.

Gleichzeitig stellt dies aber auch die wichtigste Limitation der Methodik dar, da fokale, nur sehr kurz dauernde Anfälle bzw. Anfälle mit nur geringem Unterschied in der Amplitudenhöhe von der Grundaktivität und fehlender regionaler Ausbreitung schwieriger erkennbar sind. Auch kann die Höhe der Amplituden von Medikamenten, Artefakten oder anderen klinischen Zuständen beeinflusst werden [4]. Somit sollte, soweit verfügbar, immer das zugrunde liegende Roh-EEG zur Anfallserkennung im aEEG herangezogen werden und die Diagnostik so schnell wie möglich um ein konventionelles EEG inklusive Video ergänzt werden.

\section{Fazit}

Zur verlässlicheren Anfallserkennung, zur genaueren Erfassung der "seizure burden" und zur Therapiesteuerung bei Neugeborenen mit akut symptomatischen Anfällen stellt das aEEG neben dem Goldstandard des konventionellen EEGs inklusive Video eine hilfreiche zusätzliche Methode dar.
Die Dauer und Häufigkeit der Anfälle, die Hintergrundaktivität und die Erfahrung des Befunders spielen bei der Erkennung von Anfällen im aEEG eine entscheidende Rolle.

Nur kurz dauernde, rein fokale oder sich nur wenig von der Grundaktivität unterscheidende Anfälle können damit weniger gut erfasst werden, wobei länger dauernde Anfälle, die sich regional ausbreiten, von einem erfahrenen Befunder in bis zu $85 \%$ auch mittels aEEG erkannt werden können. Da es zunehmend Daten gibt, dass die „seizure burden“ mit dem entwicklungsneurologischen Outcome korreliert und laut der aktuellen ILAE-Klassifikation eine rein klinische Diagnostik von Anfällen beim Neugeborenen nicht zu empfehlen ist, ist eine möglichst kontinuierliche elektrophysiologische Überwachung dieser Patienten anzustreben. Hier kann das aEEG einen wichtigen Beitrag leisten. Für die Zukunft ist eine automatische Anfallserkennung mittels „Anfallserkennungsalgorithmen" ein wünschenswertes Hilfsmittel in der kontinuierlichen EEG/aEEG-Ableitung.

\section{Fazit für die Praxis}

- Anfälle beim Neugeborenen sind hauptsächlich "akut symptomatisch" = Ausdruck einer zugrunde liegenden neurologischen Störung.

- Zur Diagnose von Anfällen beim Neugeborenen ist eine EEG-Ableitung unabdingbar.

- Zur genauen Erfassung der „seizure burden" (Anfallsdauer über einen bestimmten Zeitraum) ist eine kontinuierliche Langzeit-EEG-Ableitung notwendig.

- Zum Erstscreening bei klinischen Auffälligkeiten und zur Therapieüberwachung kann das aEEG - ergänzend bzw. bis zur Verfügbarkeit des konventionellen EEGs gut eingesetzt werden.

- Die einfache Handhabung direkt am Krankenbett und die reduzierte Elektrodenzahl sind wichtige Vorteile der Methode.

- Je nach Dauer, Lokalisation und Expertise des Befunders schwankt die "seizure detection rate" des aEEGs zwischen 40 und $80 \%$.

- Eine Bestätigung mittels konventionellen EEGs bzw. durch Experten ist zusätzlich notwendig.

- Automatische Seizure-Detection-Algorithmen befinden sich in Entwicklung und stellen für die Zukunft ein wünschenswertes Tool zur Anfallserkennung dar.

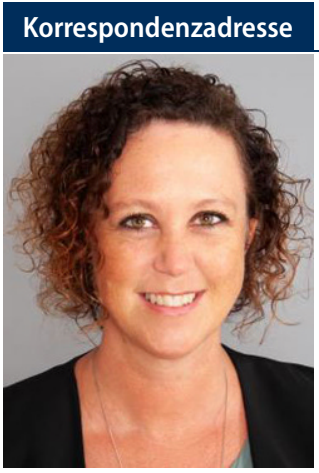

Katrin Klebermass-Schrehof

Abteilung für Kinder- und Jugendheilkunde, Division für Neonatologie, pädiatrische Intensivmedizin und Neuropädiatrie, Medizinische Universität Wien Währinger Gürtel 18-20, 1090 Wien, Österreich katrin.klebermass-schrehof@meduniwien.ac.at

Funding. Open access funding provided by Medical University of Vienna.

\section{Einhaltung ethischer Richtlinien}

Interessenkonflikt. K. Klebermass-Schrehof gibt an, Vortragshonorare und Reisekostenerstattung von Natus Medical Incorporated erhalten zu haben.

Für diesen Beitrag wurden vom Autor keine Studien an Menschen oder Tieren durchgeführt. Für die aufgeführten Studien gelten die jeweils dort angegebenen ethischen Richtlinien

Open Access. Dieser Artikel wird unter der Creative Commons Namensnennung 4.0 International Lizenz veröffentlicht, welche die Nutzung, Vervielfältigung Bearbeitung, Verbreitung und Wiedergabe in jeglichem Medium und Format erlaubt, sofern Sie den/die ursprünglichen Autor(en) und die Quelle ordnungsgemäß nennen, einen Link zur Creative Commons Lizenz beifügen und angeben, ob Änderungen vorgenommen wurden.

Die in diesem Artikel enthaltenen Bilder und sonstiges Drittmaterial unterliegen ebenfalls der genannten Creative Commons Lizenz, sofern sich aus der Abbildungslegende nichts anderes ergibt. Sofern das betreffende Material nicht unter der genannten Creative Commons Lizenz steht und die betreffende Handlung nicht nach gesetzlichen Vorschriften erlaubt ist, ist für die oben aufgeführten Weiterverwendungen des Materials die Einwilligung des jeweiligen Rechteinhabers einzuholen.

Weitere Details zur Lizenz entnehmen Sie bitte der Lizenzinformation auf http://creativecommons.org/ licenses/by/4.0/deed.de.

\section{Literatur}

1. Apers WMJ, de Vries LS, Groenendaal F, Toet MC, WeekeLC(2020)Delayin treatment of neonatal sei- 
zures: a retrospective cohort study. Neonatology 117:599-605

2. Bashir RA, Espinoza L, Vayalthrikkovil S, Buchhalter J, Irvine L, Bello-Espinosa L, Mohammad K (2016) Implementation of a neurocritical care program: improved seizure detection and decreased antiseizure medication at discharge in neonataes with hypoxic-ischemic encephalopathy. Pediatr Neurol 64:38-43

3. Bourgoin P, BarraultV, Loron Getal (2020) Interrater agreement between critical care providers for background classification and seizure detection after implementation of amplitude-integrated electroencephalography in neonates, infants, and children. J Clin Neurophysiol 37(3):259-262

4. Bruns N, Felderhoff-Mueser U, Dohna-Schwake C (2021) aEEG as useful tool for neuromonitoring in critically ill children-current evidence and knowledge gaps. Acta Paediatr 110:1132-1140

5. Chen YJ, Chiang MC, Lin JJ, Chou IJ, Wang YS, KongSS, Su IC, Chen E, Mok TJD, Lien R, Lin KL (2020) Seizures severity during rewarming can predict seizure outcomes of infants with neonatal hypoxicischemic encephalopathy following therapeutic hypothermia. Biomed J 43:285-292

6. Dilena R, Raviglione F, Cantalupo G, Cordelli DM, De Liso P, Di Capua M, Falsaperla R, Ferrari F, Fumagalli M, Lori S, Suppiej A, Tadini L, Dalla Bernardina B, Mastrangelo M, Pisani F, INNESCO Group (2021) Consensus protocol for EEG and amplitudeintegrated EEG assessment and monitoring in neonates. Clin Neurophysiol 132(4):886-903

7. Din F, Lalgudi Ganesan S, Akiyama T et al (2020) Seizure detection algorithms in critically ill children: a comparative evaluation. Crit Care Med 48(4):545-552

8. Du Pont-Thibodeau G, Sanchez SM, Jawad AF et al (2017) Seizure detection by critical care providers using amplitude-integrated electroencephalography and color density spectral array in pediatric cardiac arrest patients. Pediatr Crit Care Med 18(4):363-369

9. Glass HC, Grinspan ZM, Shellhaas RA (2018) Outcomes after acute symptomatic seizures in neonates. Semin Fetal Neonatal Med 23(3):218-222

10. Kadivar M, Moghadam EM, Shervin Badv R, Sangsari R, Saeedy M (2019) A comparison of conventional electroencephalography with amplitude-integrated EEG in detection of neonatal seizures. Med Devices (Auckl) 12:489-496

11. Karamian AGS, Wusthoff CJ (2020) How helpful is aEEG? Context and user experience matter. Am J Perinatol. https://doi.org/10.1055/s-00401721711

12. Kharoshankaya L, Stevenson NJ, Livingstone V, Murray DM, Murphy BP, Ahearne CE et al (2016) Seizure burden and neurodevelopmental outcome in neonates with hypoxicischemic encephalopathy. Dev Med Child Neurol 58:1242-1248

13. Lawrence R, Mathur A, Nguyen The Tich S, ZempelJ, Inder T (2009) A pilot study of continuous limitedchannel aEEG in term infants with encephalopathy. JPediatr 154:835-841.e1

14. Mathieson SR, Rennie JM, Livingstone V, Temko A, Pressler RM, Boylan GB (2016) In-depth performance analysis of an EEG based neonatal seizure detection algorithm. Clin Neurophysiol 127(5):2246-2256

15. Maynard D, Prior PF, Scott DF (1969) Device for continuous monitoring of cerebral activity in resuscitated patients. BrMed J 4:545-546

16. Murray DM, Boylan GB, Ali I et al (2008) Defining the gap between electrographic seizure burden, clinical expression and staff recognition of

\section{Acute symptomatic seizures in neonates and use of amplitude- integrated EEG (aEEG)}

Background: Seizures in neonates are mainly acute symptomatic seizures and a sign of an underlying neurological disorder. The diagnosis of neonatal seizures carried out according to the International League Against Epilepsy (ILAE) classification is based only on electroencephalography (EEG) and then it is described if they are accompanied by motor symptoms or not. Therefore, the simplified method of amplitude-integrated EEG (aEEG), which can be easily used for long-term monitoring directly at the bedside, has proven helpful and is not just a compensatory measure until conventional video EEG becomes available.

Objective: This manuscript discusses the value of aEEG for diagnosis and surveillance of neonatal seizures.

Results and discussion: For exact assessment of the seizure burden (duration of seizure activity within a given time frame) and for guidance of a possible treatment, the aEEG has proven value, in addition to conventional video EEG.

The duration and frequency of seizures, background activity and experience of the assessing caregiver have a significant influence on the seizure detection rate of aEEG. Only short, very focal seizures or seizures with amplitudes not significantly different from background activity cannot be easily detected by aEEG, whereby longer seizures that spread regionally can be identified by an experienced user in up to $85 \%$ using aEEG. As there are increasing data showing that the exact seizure burden correlates to neurodevelopmental outcome, a continuous neurophysiological surveillance of patients at risk is preferred. The aEEG can significantly contribute to this aim.

\section{Keywords}

Seizures in newborns - Neonatal seizures · Neurophysiological bedside monitoring · Long-term EEG monitoring

neonatal seizures. Arch Dis Child Fetal Neonatal Ed 93:F187-91

17. Pavel AM, Rennie JM, de Vries LS, Blennow M, Foran A, Shah DK, Pressler RM, Kapellou O, Dempsey EM, Mathieson SR, Pavlidis E, van Huffelen A, Livingstone V, Toet MC, Weeke LC, Finder M, Mitra S, Murray DM, Marnane WP, Boylan GB (2020) A machine-learning algorithm for neonatal seizure recognition: a multicentre, randomised, controlled trial. Lancet Child Adoles Health 4:740-749

18. Pressler RM, Cilio MR, Mizrahi EM et al (2021) The ILAE classification of seizures and the epilepsies: modification for seizures in the neonate. Position paper by the ILAE task force on neonatal seizures. Epilepsia 62(3):615-628. https://doi.org/10.1111/ epi.16815

19. Rakshasbhuvankara AA, RaoS, GhoshS, NathanEA, Nagarajan $L$ (2020) Why do neonates receive antiseizure medications? J Matern Fetal Neonata Med. https://doi.org/10.1080/14767058.2020. 1819976

20. Rennie JM, de Vries LS, Blennow M, Foran A, Shah DK, Livingstone V, van Huffelen AC, Mathieson SR, Pavlidis E, Weeke LC, Toet MC, Finder M, Pinnamaneni RM, Murray DM, Ryan AC, Marnane WP, Boylan GB (2019) Characterisation of neonatal seizures and their treatment using continuousEEG monitoring: a multicentre experience. Arch Dis Child Fetal Neonatal Ed 104:F493-F501

21. Rennie JM, Chorley G, Boylan GB, Pressler R, Nguyen Y, Hooper R (2004) Non-expert use of the cerebral function monitor for neonatal seizure detection. Arch Dis Child Fetal Neonatal Ed 89(1):F37-40
22. Rakshasbhuvankara AA, Wagha D, Athikarisamya SE, Davis J, Nathanc EA, Palumboe L, Ghoshe S, Nagarajane L, Raoa SC (2020) Inter-rater reliability of amplitude-integrated EEG for the detection of neonatal seizures. Early Hum Dev 143:105011

23. Scher MS, Alvin J, Gaus L, Minnigh B, Painter MJ (2003) Uncoupling of EEG-clinical neonatal seizures after antiepileptic drug use. Pediatr Neurol 28:277-280

24. Shellhaas RA, Chang T, Tsuchida T, Scher MS, Riviello JJ, Abend NS, Nguyen S, Wusthoff CJ, Clancy RR (2011) The American clinical neurophysiology society's guideline on continuous electroencephalography monitoring in neonates. JClin Neurophysiol 28(6):611-617

25. Shellhaas RA, Soaita Al, Clancy RR (2007) Sensitivity of amplitude-integrated electroencephalography for neonatal seizure detection. Pediatrics 120(4):770-777

26. SrinivasakumarP,Zempel J, TrivediS, WallendorfM Rao R, Smith B, Inder T, Mathur AM (2015) Treating EEG seizures in hypoxic Ischemic encephalopathy: a randomized controlled trial. Pediatrics 136(5):e1302-9

27. Stevenson NJ, Lauronen L, Vanhatalo S (2018) The effect of reducing EEG electrode number on the visual interpretation of the human expert for neonatal seizure detection. Clin Neurophysiol 129(1):265-270

28. Toet MC, Groenendaal F, Osredkar D, van Huffelen AC, de Vries LS (2005) Postneonatal epilepsy following amplitude-integrated EEG-detected neonatal seizures. Pediatr Neurol 32:241-247

29. Weeke LC, Boylan GB, Pressler RM, Hallberg B, Blennow M, Toet MC et al (2016) Role of EEG background activity, seizure burden and MRI in 
Fortbildungstipp

predicting neurodevelopmental outcome in fullterm infants with hypoxic-ischaemic encephalopathy in the era of therapeutic hypothermia. Eur J Paediatr Neurol 20:855-864

30. Wirrell EC, Armstrong EA, Osman LD et al (2001) Prolonged seizures exacerbate perinatal hypoxicischemic brain damage. Pediatr Res 50:445-454

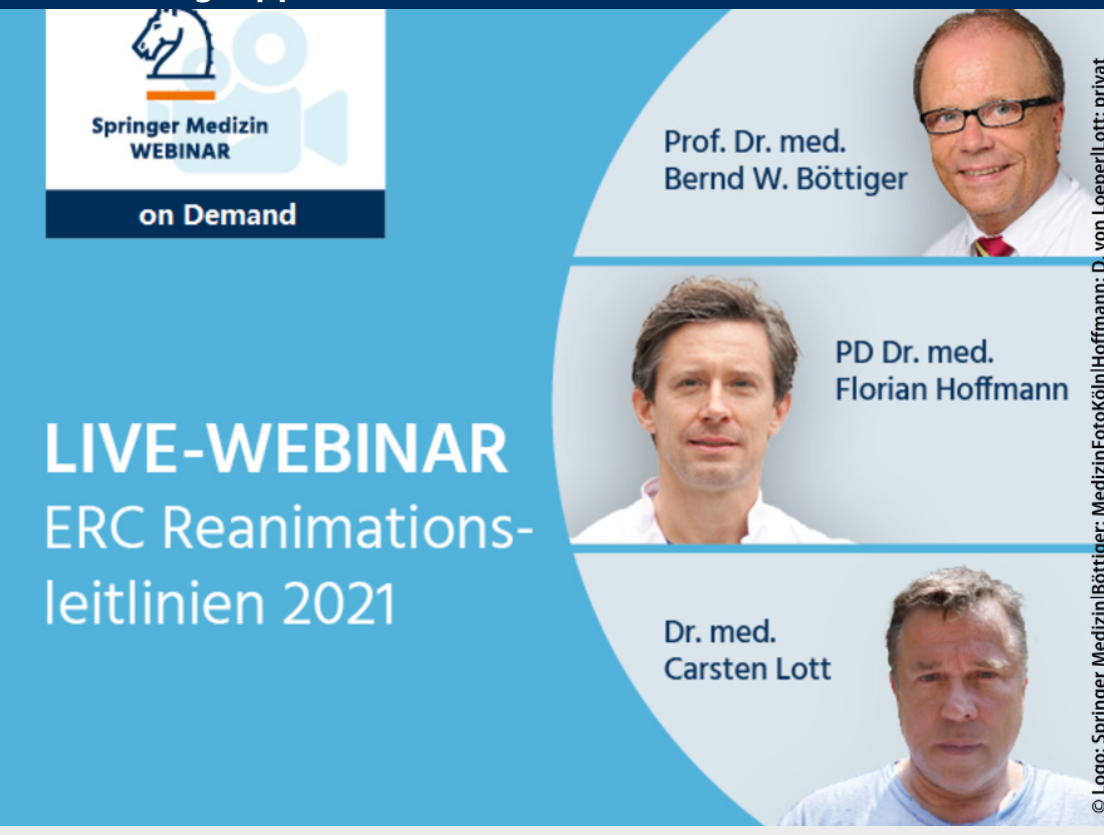

\section{Fragen zur Reanimation von Kindern?}

Ein Kind zu reanimieren ist auch für langjährige Ärtz*innen und Kolleg*innen des Rettungsdienstfachpersonals eine Herausforderungen. Oft stellen sich konkrete Fragen, die einen verunsichern. Lesen Sie hier kurz und knapp die Antworten von PD Dr. Florian Hoffmann auf einige Fragen Ihrer Kolleg*innen und die dazugehörigen Empfehlungen aus der ERC-Reanimationslinein 2021.

Das komplette Webinar zu den ERCReanimationsleitlinen

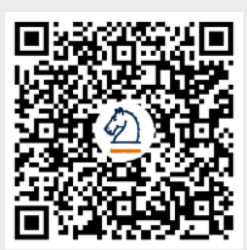

Im Webinar finden Sie die essentiellen Grundlagen der ERC-Reanimationsleitlinien zusammengefasst. Der Fokus liegt dabei auf Koronarthrombosen und Herzinfarkt und der der Renimation bei Kindern und Jugendlichen.

\section{Die Referenten:}

- Prof. Dr. B. W. Böttiger, Köln

- PD Dr. Florian Hoffman, München

- Dr. med Carsten Lott, Mainz

[URL: www.springermedizin.de/webinar-ercleitlinien]
Antworten aus 3 Fragen zum Webinar:

? Erkennt der AED anhand des Körperwiderstands eine mögliche Reduktion der Energiezahl oder werden bei einem Säugling auch $360 \mathrm{~J}$ abgegeben?

>> Bisher erkennt der AED nur, ob K-Elektroden oder E-Elektroden angeschlossen sind. In Zukunft wird aber eine Berechnung nach Körperwiderstand realisiert werden können.

? Gibt es eine Empfehlungen zum Zeitpunkt der Intubation unter CPR?

>> Die Intubation ohne Effekt auf Outcome ist sehr in den Hintergrund gerückt und nur dann durchführbar, wenn alle notwendigen Maßnahmen laufen und ein Experte für Kinderintubation anwesend ist ( $>100-150$ Intubationen).

? Gibt es eine Evidenz zur Verwendung von Erwachsenen-Masken bei Kindern, im Sinne einer Full-Face-Alternative, falls kein Kinderbeutel verfügbar ist? >> Es gibt keine Evidenz aber Erfahrungen mit Drehen der Maske um 180 Grad, mit Einmalmasken mit aufblasbarem Rand kann auch mit Erwachsenen-Masken zumeist eine gute Ventilation erreicht werden. 